\title{
Low-Noise Readout of TES Detectors with Baseband Feedback Frequency Domain Multiplexing
}

\author{
R. den Hartog • M.D. Audley • J. Beyer • D. Boersma • M. Bruijn • L. Gottardi • \\ H. Hoevers • R. Hou • G. Keizer • P. Khosropanah • M. Kiviranta • P. de Korte • \\ J. van der Kuur • B.-J. van Leeuwen • A.C.T. Nieuwenhuizen • P. van Winden
}

Received: 21 August 2011 / Accepted: 24 January 2012 / Published online: 9 February 2012

(C) The Author(s) 2012. This article is published with open access at Springerlink.com

\begin{abstract}
SRON is developing an electronic read-out system for an array of transition edge sensors (TES) based on the techniques of frequency domain multiplexing (FDM) and base-band feedback (BBFB). The astronomical applications of our system are the read-out of soft X-ray micro-calorimeters in a potential instrument on the European X-ray mission-under-study Athena and far-IR bolometers for the SAFARI instrument on the Japanese mission SPICA. In this paper we demonstrate the simultaneous locking of up to 51 BBFB loops. While locked, the in-band read-out noise of the loops is shown to reach the $10 \mathrm{pA} / \sqrt{\mathrm{Hz}}$ level required for these missions.
\end{abstract}

Keywords Transition edge sensors · Multiplexed read-out · Bolometers · Microcalorimeters $\cdot$ Frequency domain multiplexing

\section{Introduction}

FDM is one of the systems that allow the multiplexed read-out of multiple TES detectors using a single SQUID-based pre-amplifier, thus significantly reducing the heatload on the array from external wiring [1]. It has already found application in several

R. den Hartog $(\varangle)$ · D. Boersma · M. Bruijn · L. Gottardi · H. Hoevers · R. Hou · G. Keizer ·

P. Khosropanah · P. de Korte · J. van der Kuur · B.-J. van Leeuwen · A.C.T. Nieuwenhuizen · P. van Winden

SRON Netherlands Institute for Space Research, Sorbonnelaan 2, 3584 CA Utrecht, The Netherlands e-mail: r.h.den.hartog@sron.nl

M.D. Audley

P.O. Box 800, 9700 AV Groningen, The Netherlands

J. Beyer

PTB, Abbestrasse 2-12, Berlin, 10587, Germany

M. Kiviranta

VTT, Tietotie 3, 02110 Espoo, Finland 
Table 1 Requirements on the FDM read-out system for the Athena XMS and Spica Safari instruments

\begin{tabular}{lll}
\hline & Athena XMS & SPICA Safari \\
\hline \# pixels / channel & $16-32$ & 160 \\
total \# pixels & 1024 & $4100-6400$ \\
read-out mode & triggered & continuous \\
resolution & $2.5 \mathrm{eV}$ FWHM at $6 \mathrm{keV}$ & $2-4 \times 10^{-19} \mathrm{~W} / \sqrt{\mathrm{Hz}}$ \\
& $\left(\equiv 10^{-17} \mathrm{~W} / \sqrt{\mathrm{Hz}} \mathrm{NEP}\right)$ & optical NEP \\
noise at SQUID input & $<14 \mathrm{pA} / \sqrt{\mathrm{Hz}}$ & $<10.5 \mathrm{pA} / \sqrt{\mathrm{Hz}}$ \\
signal bandwidth & $5 \mathrm{kHz}$ & $<60 \mathrm{~Hz}$ \\
detector power plateau & $10 \mathrm{pW}$ & $10 \mathrm{fW}$ \\
crosstalk & $<2 \times 10^{-4}$ & $<1 \times 10^{-4} \mathrm{a}$ \\
dynamic range density & $<6.4 \times 10^{5} \sqrt{\mathrm{Hz}}$ & $<7 \times 10^{4} \sqrt{\mathrm{Hz}}$ \\
carrier frequency range & $1-4 \mathrm{MHz}$ & $1-3 \mathrm{MHz}$ \\
carrier freq. spacing & $100 \mathrm{kHz}$ & $12.5 \mathrm{kHz}$ \\
carrier freq. accuracy & $\pm 10 \mathrm{kHz}$ & $\pm 1.2 \mathrm{kHz}$ \\
LC filter quality $Q$ & $1400-5600$ & $>7000$ \\
\hline
\end{tabular}

${ }^{\mathrm{a}}$ Crosstalk requirements for $1^{\text {st }}, 2^{\text {nd }}, 3^{\text {rd }}$ and $4^{\text {th }}$ neighbor pixels are less stringent

ground-based systems, mainly in bolometer read-out for Cosmic Microwave Background observations [2-4]. FDM is based on the amplitude modulation of a carrier signal with the signal detected by the TES detector [5, 6]. BBFB [7-9] attempts to cancel the error signal in the SQUID sum-point, improving the signal bandwidth and the loop gain. For the Athena XMS instrument the goal is to read out 32 pixels per SQUID in the frequency range from 1 to $4 \mathrm{MHz}$, while for the Safari instrument on the Spica mission 160 TES pixels are required in the frequency range from 1 to $3 \mathrm{MHz}$, owing to the smaller signal bandwidth of the bolometers and the more stringent demands on dissipated power. Further requirements on the read-out systems for both missions are listed in Table 1. From a read-out point of view, both sets of requirements are very similar, allowing at least partial parallel development tracks. In particular, the required noise levels are comparable, in the range of $10-14 \mathrm{pA} / \sqrt{\mathrm{Hz}}$.

\section{Experimental Set-UP}

A generic schematic for the system under development at SRON is shown in Fig. 1. The experiments described in this paper involved two different set-ups. The first is a repetition of a previous set-up used in the IXO XMS development activity [10]. Several improvements were made to reduce the read-out noise, including an upgrade of the SQUID to the PTB C5 series with bias-current feedback [11], and an LNA with lower noise. The new $5 \times 5$ detector array with slower TES pixels to reduce the dynamic range demands on the SQUID turned out to have pixels with $T_{\mathrm{C}}$ 's barely above the ADR base temperature, so it was operated only in normal state. The second set-up is a first step towards multiplexed bolometer read-out taken in the SAFARI development track. Shown in Fig. 2 is a bracket with (counter clockwise, starting upper 


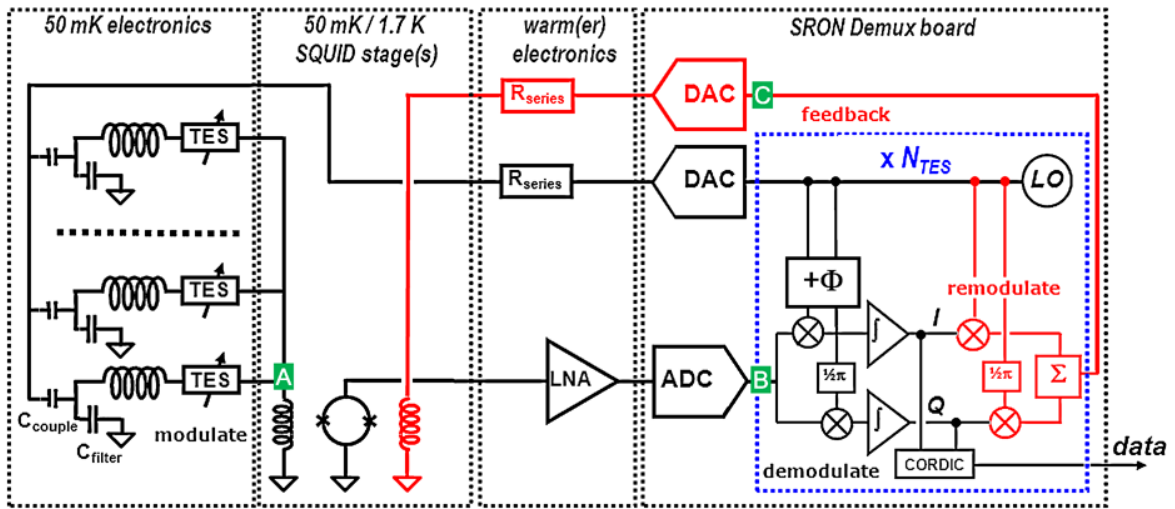

Fig. 1 (Color online) Schematic of the FDM BBFB system. Indicated are $(A)$ reference point for noise measurements, $(B)$ measurement point for open-loop noise, and $(C)$ measurement point for closed-loop noise

Fig. 2 (Color online) Safari cold head for FDM bolometer read-out

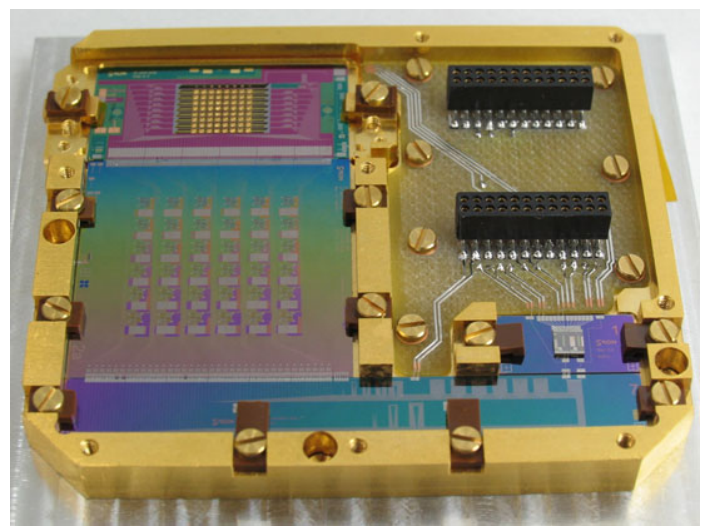

left-corner) a 64-pixel detector array, matching LC filter array [12], interconnection chip, and the same PTB C5 SQUID on fan-out chip. The bracket fits in a light-tight housing. The bolometer array showed a large spread in $T_{\mathrm{C}}$ 's with the lowest again barely above the ADR base temperature, so it was also operated only in normal state. This is sufficient for a proper verification of the noise of the read-out system, as the noise from the TESs is lower when they are in normal state, compensating the noise increase due to an elevated bath temperature.

\section{Measurements}

The attempts to reduce the read-out noise have paid off, as can be seen in Fig. 3. In the left panel the open-loop noise, measured at the input of the ADC (point B, Fig. 1) is shown. The indicated noise level, referred to the SQUID input (point A, Fig. 1), corresponds to $10 \mathrm{pA} / \sqrt{\mathrm{Hz}}$, and it is clear that the actual noise level stays below this line over the entire relevant frequency range. This implies a factor 5 improvement 

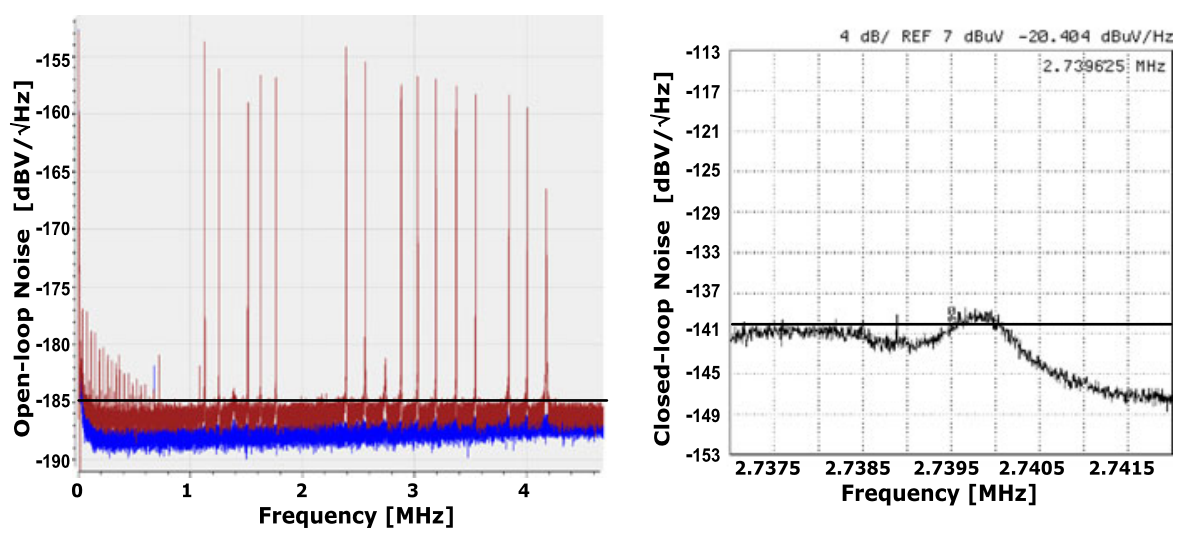

Fig. 3 (Color online) Left: standard open-loop noise measurement with SQUID biased in set point (light) and not biased (dark). Right: in-band noise measured in one channel with 16 channels in BBFB loop, and 15 carriers switched on. The horizontal lines indicate the $10 \mathrm{pA} / \sqrt{\mathrm{Hz}}$ level

with respect to previous experiments [10], and indicates that the read-out noise requirements in Table 1 are feasible. The difference between the two noise levels in the left panel is the signal picked up by the SQUID in its set point, and consists at offresonance frequencies mainly of SQUID wide-band noise. The root square difference amounts to $4.3 \mathrm{pA} / \sqrt{\mathrm{Hz}}$ or $0.19 \mu \Phi_{0} / \sqrt{\mathrm{Hz}}$ (at $1 \mathrm{MHz}$ ), which is consistent with PTB specifications.

Missing from these open-loop noise measurements is the contribution from the feedback path and the actual confirmation that the noise in the signal band around the carrier also stays below the required level. The right panel shows therefore a measurement of the noise in a $\pm 25 \mathrm{kHz}$ band around one of the demodulation frequencies (which correspond usually one-on-one to LC resonance frequencies), taken at the location of the DAC that generates the baseband feedback signal (point C, Fig. 1). The bath temperature in this measurement was set to $100 \mathrm{mK}$, which is above the $T_{\mathrm{C}}$ of the TES detectors. Since then no carriers are necessary to keep the detectors in a setpoint, it was possible to reduce them, either individually or simultaneously, to zero, after the BBFB loops were closed. The peak in the noise spectra around the resonance frequency contains information about the in-band read-out noise, which is equal to the peak level corrected for the Johnson noise of the resistor. The normal resistance was measured to be $66 \pm 3 \mathrm{~m} \Omega$ in an open-loop $Q$ measurement. Taking into account a $3 \mathrm{mK}$ uncertainty in the bath temperature, this results in an estimated Johnson noise of $9.0 \pm 0.5 \mathrm{pA} / \sqrt{\mathrm{Hz}}$. In Fig. 3 the derived level of the noise peak is $12.4 \mathrm{pA} / \sqrt{\mathrm{Hz}}$, which corresponds, after correction for the Johnson noise, to a read-out noise level of $\sim 8.5 \mathrm{pA} / \sqrt{\mathrm{Hz}}$ at the SQUID input. It confirms the result obtained in the open-loop noise measurements.

Figure 4 illustrates a disadvantage of the current LC filter design, which combines a couple capacitor and a filter capacitor to form a 1000:1 AC voltage divider (see Fig. 1). For this ratio, the couple capacitor has to be rather small, making it vulnerable to ESD events. When shorted, noise on the AC bias line leaks in over a wide bandwidth. Figure 3 was obtained after the wirebonds to the faulty channel 

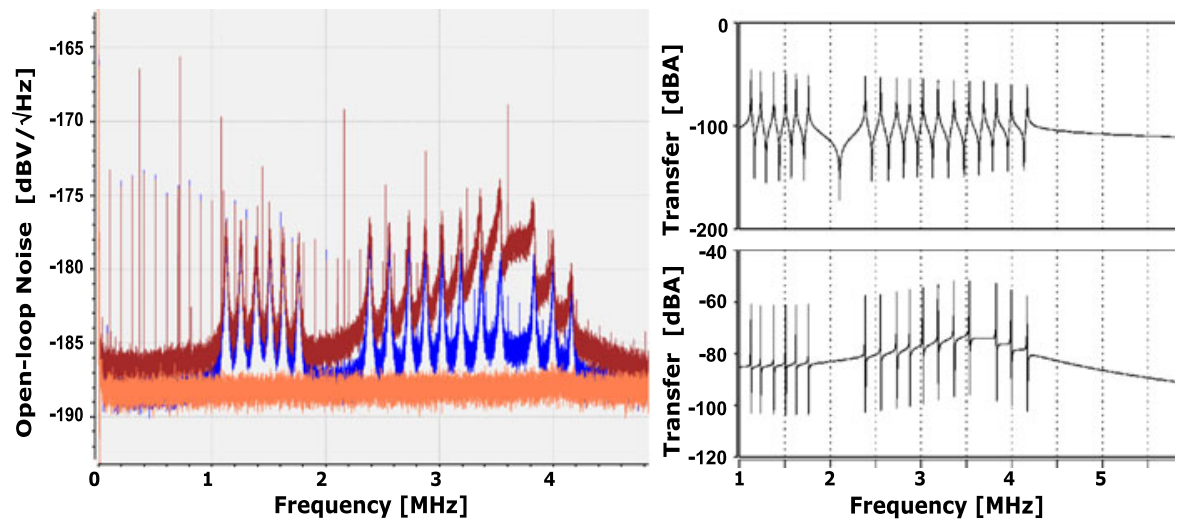

Fig. 4 (Color online) Left: open-loop noise with a shorted couple capacitor in the circuit at $3.7 \mathrm{MHz}$ for different values of the series resistance. Right top: LT-SPICE simulation of expected circuit transfer function, bottom: simulated transfer function with shorted couple capacitor
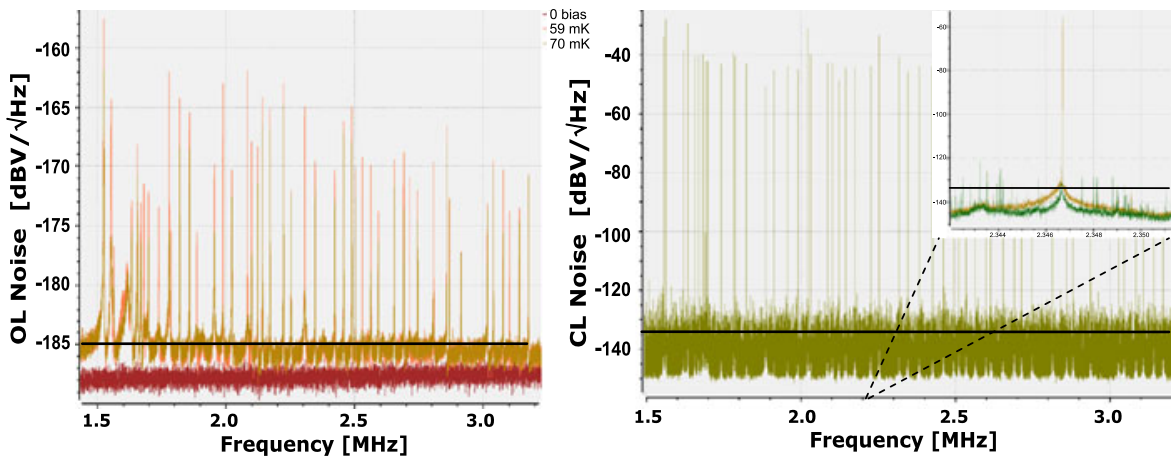

Fig. 5 (Color online) Noise measurements on the new 64 pixel set-up shown in Fig. 2. Horizontal lines indicate the $10 \mathrm{pA} / \sqrt{\mathrm{Hz}}$ level. Left: open-loop noise measurements with SQUID in set-point and un-biased. Right: noise measurement at highest $T_{\text {bath }}$ with 51 carriers in closed BBFB loop. Inset shows blow up around one frequency with and without $\mathrm{AC}$ carrier

were physically removed from the chip. Future LC filter chip designs will use a more robust, multi-stage AC bias voltage division.

Finally, Fig. 5 shows the open- and closed-loop measurements for the 64 pixel bolometer set-up shown in Fig. 2. Both the bolometer and LC filter array suffered from some yield problems, leaving only 54 resonances intact of which 51 could be simultaneously locked in BBFB loops. Like in the previous experiment the TES detectors were driven into the normal state by an elevated bath temperature of $120 \mathrm{mK}$. An excursion in the open-loop noise level seems present near $1.6 \mathrm{MHz}$, similar to that in Fig. 4, indicating a defect couple capacitor. At higher frequencies the noise appears to stay below the $10 \mathrm{pA} / \sqrt{\mathrm{Hz}}$ level, which is again confirmed by the closed loop noise. The noise peak in the inset of the right panel corresponds to $12.8 \mathrm{pA} / \sqrt{\mathrm{Hz}}$. The normal resistance of this detector is $160 \mathrm{~m} \Omega$ (with an estimated uncertainty of $10 \mathrm{~m} \Omega$ ), which gives an estimate of the Johnson noise of $6.0 \pm 0.5 \mathrm{pA} / \sqrt{\mathrm{Hz}}$. Correcting for 
the Johnson noise yields a read-out noise of $\sim 11.5 \mathrm{pA} / \sqrt{\mathrm{Hz}}$. This demonstrates that multiplexing does not degrade the read-out noise.

This experiment is just a first step towards the Safari instrument. Further steps, to be taken in the coming months, include:

- Fabrication of LC filter chips with more robust voltage division,

- Multiplexed low-noise read-out of TES bolometers in a set-point,

- Demonstration of low optical NEPs in multiplexed read-out.

Open Access This article is distributed under the terms of the Creative Commons Attribution License which permits any use, distribution, and reproduction in any medium, provided the original author(s) and the source are credited.

\section{References}

1. K. Irwin, AIP Conf. Proc. 1185, 229 (2009)

2. T.M. Lanting et al., Appl. Phys. Lett. 86, 112511 (2005)

3. A.T. Lee, Nucl. Instrum. Methods A 559, 786 (2006)

4. M. Dobbs, M. Lueker et al., arXiv:1112.4215 [astro-ph] (2011)

5. J. Yoon et al., Appl. Phys. Lett. 78, 371 (2001)

6. M. Kiviranta, H. Seppä, J. van der Kuur, P. de Korte, AIP Conf. Proc. 605, 295 (2002)

7. J. van der Kuur et al., AIP Conf. Proc. 1185, 245 (2009)

8. R. den Hartog et al., AIP Conf. Proc. 1185, 261 (2009)

9. Y. Takei et al., Supercond. Sci. Technol. 22, 114008 (2009)

10. R. den Hartog et al., IEEE Trans. Appl. Supercond. 21(3), 289 (2011)

11. D. Drung, J. Beyer, M. Peters, J.-H. Storm, T. Schurig, IEEE Trans. Appl. Supercond. 19(3), 772 (2009)

12. M.P. Bruijn et al., These Proceedings. J. Low Temp. Phys. (2012). doi:10.1007/s10909-011-0422-5 